\title{
EDUCACIÓN PARVULARIA CHILENA: EFECTOS POR GÉNERO Y AÑOS DE PARTICIPACIÓN ${ }^{1}$
}

\author{
Alejandra Cortázar ${ }^{2}$ \\ Constanza Vielma ${ }^{3}$
}

RESUMEN

El presente estudio tiene como objetivo estimar el efecto de la educación parvularia pública chilena en los resultados académicos de los niños en el Sistema de Medición de la Calidad de la Educación (Simce) de cuarto básico. El estudio busca entender si estos efectos varían en función del género del niño y del número de años de participación en la educación parvularia. Mediante la metodología de Propensity Score Matching se encontró que asistir a la educación parvularia pública afecta positivamente los resultados académicos en Matemáticas y Lenguaje en cuarto básico. Este efecto fue mayor para los niños que para las niñas, y para los que asistieron a Nivel Transición 1.

Palabras clave: desempeño académico, educación parvularia, estimación impacto, género.

\section{CHILEAN EARLY CHILDHOOD EDUCATION: GENDER AND YEARS OF ATTENDANCE EFFECTS}

\begin{abstract}
The present study aims to estimate the effect of Chilean public early childhood education on the academic outcomes of 4th grade children on the Education Quality Measurement System (Simce, for its abbreviation in Spanish). The study seeks to understand if these effects vary according to the child's gender and by the number of years of participation in early childhood education. Through Propensity Score Matching, it is observed that attending public early childhood education positively affects $4^{\text {th }}$ grade academic results in Mathematics and Language. The early childhood education effect was greater for boys than for girls, and for those who assisted Transition Level 1.
\end{abstract}

Keywords: academic performance, early childhood education, effect estimation, gender.

1 En este estudio los autores utilizaron bases de datos de la Agencia de la Calidad de la Educación, Fundación Integra y la Junta Nacional de Jardines Infantiles. Los autores están agradecidos de dichas agencias. El estudio es responsabilidad única de los autores.

2 Centro de Estudios de la Primera Infancia, Santiago, Chile.

Contacto: alejandracortazar@gmail.com

3 Centro de Estudios, Ministerio de Educación, Santiago, Chile. Contacto: cpvielma@gmail.com 


\section{Introducción}

En las últimas dos décadas se ha considerado a la primera infancia como un período de gran relevancia en el desarrollo humano. Estudios revelan que durante esta etapa, el cerebro se desarrolla a un ritmo más rápido debido a una sobreproducción sináptica (Shonkoff \& Phillips, 2000); al mismo tiempo que se abren ventanas de oportunidades para el desarrollo del lenguaje y de las funciones cognitivas (Shore, 1997). Las primeras experiencias, además, son determinantes en la percepción que tienen los niños de sí mismos, las relaciones con los demás y la capacidad de estos de confiar en otros (Siegel, 1999). Múltiples estudios muestran cómo la educación parvularia de calidad puede tener efectos positivos en el corto y en el largo plazo. Particularmente, estudios de impacto de programas internacionales han demostrado un efecto positivo en los resultados académicos de los niños y en su adaptación socioemocional durante la adolescencia y adultez (Barnett, 1985, 1995; Ramey \& Ramey, 2006).

La educación parvularia de calidad ha demostrado tener efectos positivos en las personas, a la vez que resulta rentable a nivel social (Belfield, Nores, Barnett \& Schweinhart, 2006; Heckman, 2000). Esta evidencia ha llevado a países alrededor del mundo a poner mayor énfasis en expandir el acceso a educación parvularia y a mejorar la calidad de sus programas. Dado el énfasis actual en políticas para la educación inicial, evaluar los efectos a largo plazo de este tipo de programas en el logro académico es cada vez más importante. Los resultados positivos fortalecen el argumento de invertir fondos públicos en programas de educación inicial y debilitan aquellos que van en la dirección contraria (Magnuson, Ruhm \& Waldfogel, 2007).

Si bien en Chile existe evidencia acerca del efecto positivo que tiene asistir a la educación inicial sobre el logro académico posterior (Contreras, Herrera y Leyton 2007), hay escasa investigación en torno a caracterizar las particularidades de estos efectos. En un país como Chile, donde existe una baja cobertura de la educación parvularia para niños menores de tres años ${ }^{4}(18 \%)$, a la vez que es el país de

4 Según los datos de la Encuesta CASEN 2011. 
la Organización para la Cooperación y el Desarrollo Económicos, OCDE, que posee la brecha de género más amplia en los resultados de la prueba PISA (OECD, 2013); resulta urgente indagar en el efecto de la educación parvularia, de modo de incluir factores como los años de permanencia en los programas y la perspectiva de género.

Con el fin de abordar este vacío investigativo, el presente estudio busca estimar el efecto de asistir a la educación parvularia pública en Chile en los puntajes Simce de $4^{\circ}$ año básico (2008); e indagar en los posibles efectos diferenciados según género y años de participación. Se espera que los resultados de este estudio enriquezcan la discusión acerca de la urgencia e idoneidad de las políticas públicas para la primera infancia.

\section{Efectos académicos de la educación inicial en el largo plazo: evidencia internacional}

Un abundante cuerpo de investigación acerca de educación parvularia en Estados Unidos muestra que esta fomenta el logro académico en el largo plazo. Sin embargo, los resultados varían dependiendo de la calidad de los programas y del diseño de cada estudio (Barnett, 2008; Camilli, Vargas, Ryan \& Barnett, 2010; Nelson, Westhue \& MacLeod, 2003). Un metaanálisis sobre el efecto de los programas de educación inicial (Camilli et al., 2010) analizó 123 estudios y encontró que el efecto promedio en los resultados cognitivos oscilaba entre 0,12 y 0,48 desviaciones estándar.

Los estudios que han encontrado efectos más grandes en el largo plazo ${ }^{5}$ usualmente han sido las investigaciones acerca de los programas modelo ${ }^{6}$, los que comparten algunas características: están dirigidos a niños de bajos recursos, son programas con altos indicadores de calidad (trabajan con grupos pequeños), y cuentan con una proporción alta de profesores-niños y educadores bien entrenados (Barnett, 1995, 2008). Los dos programas modelo más

Se considera largo plazo cuando los niños son evaluados después de cinco o más años, luego de terminar el programa de educación inicial.

6 Los programas modelo son aquellos diseñados como parte de una investigación y que son sometidos a un diseño de evaluación experimental o cuasiexperimental. 
conocidos de Estados Unidos son el Abecedarian y el Perry Preschool. Ambos lograron diferencias significativas en el logro en Lenguaje y Matemáticas para los participantes en el corto y largo plazo (Besharov, Germanis, Higney \& Call, 2011; Campbell, Pungello, Miller-Johnson, Burchinal \& Ramey, 2001).

Estudios de observación longitudinal como los del Early Childhood Longitudinal Study Kindergarten Class (ECLS-K) y el Early Child Care Study (National Institute of Child Health and Human Development Network, NICHD, 2005) también han encontrado efectos académicos positivos en el largo plazo para estudiantes que asistieron a educación inicial, aunque estos son más pequeños que los reportados por los programas modelo. Estos estudios consideran la asistencia a programas a gran escala con una calidad estructural menor. Magnuson, Meyers, Ruhm y Waldfogel (2004), usando datos del ECLS-K, hallaron que los niños que participaron en la educación preescolar obtuvieron mejores puntajes en lectura en primer y tercer grado que los niños que fueron cuidados en su casa. El Early Child Care Study (NICHD, 2005) también encontró que la educación inicial tenía efecto sobre las habilidades cognitivas en tercero básico, según la prueba Woodcock-Johnson.

En cuanto al contexto de América Latina, diversos estudios en torno a programas de educación inicial han hallado efectos positivos en el desarrollo cognitivo, académico, emocional y nutricional del niño (Nores \& Barnett, 2010). Por ejemplo, Berlinski, Galiani y Manacorda (2008) evidenciaron que la participación en educación parvularia en Uruguay aumentaba las probabilidades de mantenerse en la escuela a los 15 años (1,03 años más de escolaridad que el grupo control). En Argentina, Berlinski, Galiani y Gertles (2006) encontraron que la asistencia de un año a la educación parvularia tenía un efecto positivo de 0,23 desviaciones estándares en los resultados de pruebas estandarizadas en el tercer grado. De la misma forma, la evaluación de la asistencia a la educación parvularia en Cali (Colombia), enfocada en niños de bajos ingresos y deficiencias nutricionales, mostró los efectos positivos del programa en las tasas de repitencia a los 10 años (McKay \& McKay, 1983). 
La investigación tanto acerca de programas modelo como de programas a gran escala en Estados Unidos y Latinoamérica sugiere, entonces, que la educación inicial puede tener un impacto positivo en el largo plazo. Aunque no es posible extrapolar estos resultados a los programas chilenos de educación parvularia, estas investigaciones proveen de un marco de referencia en torno al cual se puede establecer el rango de efectos esperables para los programas de educación parvularia en Chile.

\section{Efectos académicos de la educación inicial a largo plazo: el caso chileno}

\subsection{El contexto de la educación parvularia en Chile}

La educación inicial en Chile se divide en: Sala Cuna (tres meses a un año 11 meses), Medios (dos a tres años 11 meses) y de Transición (cuatro a cinco años 11 meses). Los niveles de Transición se dividen entre Nivel Transición 1 y Nivel Transición 2. Los programas de educación parvularia pueden ser financiados pública o privadamente. Los programas públicos entregan educación gratis de tiempo completo.

Desde la década de los noventa, Chile ha aumentado sustantivamente el acceso a la educación parvularia para niños de familias vulnerables y, al mismo tiempo, ha intentado mejorar la calidad de esos programas (Ministerio de Educación, Mineduc, 2013). La meta principal ha sido reducir las desigualdades educativas y disminuir la brecha de logro entre niños vulnerables y aquellos de mayores recursos (Pacheco, Elacqua y Brunner, 2005). Aunque la cobertura de educación inicial para niños de familias vulnerables aumentó de un 12,4\% en 1990 a un 32,3\% en 2009, existe poca investigación que evalúe el efecto que estos programas han tenido sobre los resultados académicos.

\subsection{Evidencia nacional de los efectos de la educación parvularia en Chile}

Usando datos del Simce, algunos estudios han medido la relación entre educación parvularia y logro académico. El Simce es 
administrado por el Ministerio de Educación y evalúa anualmente el logro académico en Matemáticas y Lenguaje y Comunicación para todos los estudiantes chilenos en segundo, cuarto, sexto y octavo año básico, y segundo y tercer año medio.

Reveco y Mella (1999) indagaron en el impacto del Nivel Transición 2 en los resultados del Simce en octavo básico, descubriendo que los niños que asistieron a este nivel obtuvieron puntajes más altos (3,4 puntos promedio) que aquellos que no lo hicieron. En otro estudio, Eyzaguirre y Le Foulon (2001) observaron el impacto del Nivel Transición 2 en los resultados de cuarto básico, encontrando que los niños que asistieron a Nivel Transición 2 obtuvieron en promedio 3,3 puntos más que aquellos que no lo hicieron. En el estudio de Contreras y colaboradores (2007) se evaluó el impacto de los Niveles Transición 1 y 2 en el logro académico en segundo año medio. Los autores encontraron que los estudiantes que asistieron a estos niveles obtuvieron alrededor de 6 a 8 puntos más en las pruebas de Lenguaje y Matemáticas, respectivamente, que aquellos que no lo hicieron.

Hernández (2006) también buscó estimar el efecto de la asistencia a Nivel Transición 1 y Nivel Transición 2 en el Simce de cuarto año básico (2002). Al igual que Contreras y colegas (2007) usó Propensity Score Matching con el objetivo de corregir por el sesgo de selección ${ }^{7}$. Hernández encontró un efecto positivo $(0,16$ de desviación estándar) de la asistencia a Nivel Transición 1. Finalmente, Bucarey, Urzúa y Ugarte (2014) analizaron los resultados del Simce de cuarto año básico entre los años 2008 a 2011 y estimaron el impacto de la educación parvularia usando Mínimos Cuadrados Ordinarios, variables instrumentales y una metodología de Propensity Score Matching. Los resultados indican que la asistencia a la educación parvularia tuvo un efecto positivo en la cohorte que rindió el Simce en el año 2008. Sin embargo, estos efectos desaparecerían en cohortes más recientes (2009-2011).

El sesgo de selección se refiere a que los participantes, por el hecho de participar, son diferentes de los no participantes. El Propensity Score Matching busca reducir este sesgo al comparar a individuos que comparten características de pretratamiento. 
Como se observa, la gran mayoría de estos estudios no controlaron por la asistencia a otros niveles de educación parvularia antes de los niveles de Transición; tampoco controlaron por tipo de financiamiento (público o privado). Adicionalmente, estos trabajos descansan sobre el autorreporte de los padres acerca de la participación de sus hijos en la educación inicial, a diferencia del presente estudio que utiliza bases administrativas de los proveedores. Por otro lado, es importante mencionar que los efectos encontrados por Reveco y Mella (1999) y Eyzaguirre y Le Foulon (2001) fueron muy pequeños, tomando en cuenta que las diferencias en el Simce son estadísticamente significativas desde los tres puntos hacia arriba. Finalmente, ninguno de estos estudios indagó en los efectos de la educación parvularia según género.

\section{3. ¿Quiénes se benefician más de la educación parvularia?}

\subsection{Género}

Si bien existe vasta evidencia de que la educación parvularia puede tener efectos positivos en el largo plazo para niños y niñas, hay poca evidencia respecto de si este efecto es igual o varía en función del género. Pocos estudios han evaluado el impacto diferencial de la educación parvularia por género, teniendo estos estudios resultados no concluyentes. Por ejemplo, Anderson (2007) analizó los datos del Abecedarian y Perry Preschool y encontró que las mujeres se beneficiaban más que los hombres de estos programas, especialmente en lo referente al número de años de educación inicial que alcanzaban a completar. Recientemente García, Heckman y Ziff (2017) encontraron que los niños eran más sensibles a la calidad del programa de educación parvularia que las niñas, beneficiándose ellas solo de los de alta calidad.

Un antecedente para entender las posibles diferencias de género en este tema son los estudios que indican que las niñas ingresarían a la educación parvularia con conocimientos académicos más avanzados que los niños (Ready, LeGerfo, Burkman \& Lee, 2005; Soderman, Chhikara \& Kuo, 1999). A nivel nacional no se cuenta con 
evidencia acerca del desarrollo de los niños y las niñas al momento de ingresar al sistema escolar de una manera comparativa.

Conocer si hay efectos diferenciados de la educación inicial en torno al logro posterior de niños y niñas es particularmente relevante para Chile, donde hay evidencia de una importante brecha de género en los resultados de los estudiantes en pruebas estandarizadas, tanto nacionales como internacionales (OECD, 2013). Por ejemplo, Chile es el país donde la brecha de género en la prueba PISA de Matemáticas es de las más pronunciadas: esta llega a casi 20 puntos a favor de los hombres; y donde la brecha de Lenguaje es la más pequeña, beneficiando levemente a las mujeres.

Estudiar las oportunidades que ofrece la educación parvularia para niños y niñas es particularmente relevante en este momento, cuando hay una preocupación a nivel mundial por ofrecer igualdad de oportunidades educativas, en particular, si el género tiene un rol importante en el aprendizaje de los estudiantes (United Nations Educational, Scientific and Cultural Organization, Unesco, 2010).

\subsection{Años en la educación inicial}

Abundante evidencia internacional ha indagado en el efecto de la cantidad de tiempo (años o meses) en que se asiste a la educación parvularia sobre los resultados posteriores de estos niños. Sin embargo, no se ha llegado a un consenso: Anderson (1992) encontró efectos positivos en un ingreso temprano a la educación inicial sueca en la competencia cognitiva y socioemocional de los niños a las edades de 8 y 13 años. En la misma línea Caughy, DiPietro y Strobino (1994) observaron mayores puntajes en lectura y matemáticas a los cinco y seis años, según el Peabody Individual Achievement Test entre los niños que iniciaron su asistencia a day-care antes de su primer cumpleaños. Sin embargo, este factor se mantuvo positivo solo para niños de hogares en situación de pobreza. Finalmente, Vandell y Corasaniti (1990) hallaron que en un contexto de estándares mínimos de educación parvularia en Suecia, los niños con mayor cantidad de años de preescolar demostraron a los ocho años, peores notas y resultados en pruebas estandarizadas, relaciones negativas con sus 
pares y fueron catalogados como los más indisciplinados por parte de sus profesores.

\section{Objetivos}

El presente estudio busca estimar los efectos en el largo plazo de la educación parvularia pública en Chile sobre el logro académico de los estudiantes, su relación con el género de los niños y con el número de años de participación en ella. De esta manera las preguntas de investigación son las siguientes:

- ¿Existe un impacto en el largo plazo de la asistencia a la educación parvularia pública chilena sobre los resultados académicos de los niños en pruebas estandarizadas en cuarto básico?

- ¿Hay diferencias en el efecto a largo plazo de la educación parvularia sobre los resultados académicos según género y número de años en la educación parvularia?

\section{Metodología}

El presente estudio comparó los resultados en cuarto año básico de los estudiantes que asistieron a la educación parvularia pública chilena con los de los estudiantes que no asistieron a la educación parvularia antes de Nivel Transición 2, pero que compartían características socioeconómicas y demográficas similares. Para propósitos de este estudio, se definió la participación en la educación parvularia como la asistencia de, por lo menos, un año en cualquiera de los niveles correspondientes al rango de los dos a cinco años (Nivel Medio Menor a Nivel Transición 1). El haber participado en Sala Cuna no fue incluido, porque no existían datos digitales confiables al respecto. La asistencia a Nivel Transición 2 también fue excluida por existir acceso universal.

La evidencia de un sesgo de selección en la participación en la educación parvularia (Kagan, 2009) generó la necesidad de usar Propensity Score Matching (PSM). Este método fue utilizado para estimar el efecto de la educación parvularia pública en el logro académico a través de: a) parear niños que asistieron a la educación 
parvularia $(n=31.947)$ con aquellos que no lo hicieron $(n=54.571)$ de acuerdo con su probabilidad de asistir a la educación parvularia pública (Propensity Score o el puntaje de probabilidad); y b) comparar el puntaje obtenido en las pruebas Simce 2008.

\subsection{Muestra}

La prueba Simce del año 2008 evaluó al 95\% de los estudiantes de cuarto año básico del país (245.607) en Lenguaje y Comunicación, y Matemáticas. De estos, los 31.947 niños que asistieron a la educación parvularia pública antes de ingresar a nivel de Transición 2 corresponden el "grupo de tratamiento", mientras que aquellos que no participaron de ningún programa (ni privado ni público) de educación parvularia conforman el "grupo de control". Las Tablas 1 y 2 describen las principales características de la muestra:

Tabla 1

Principales características de la muestra

\begin{tabular}{lcccc}
\hline & & Asistió a Ed. Parvularia (\%) & No asistió (\%) & N \\
\hline \multirow{2}{*}{ Muestra total } & & 36,9 & 63,1 & 86,518 \\
\multirow{2}{*}{ Género } & Niñas & 37,2 & 62,8 & 43,633 \\
& Niños & 36,6 & 63,4 & 42,895 \\
\hline
\end{tabular}

Tabla 2

Muestra según años de participación

\begin{tabular}{lccc}
\hline & & $\%$ & $N$ \\
\hline \multirow{2}{*}{$\begin{array}{l}\text { Años de participación } \\
\text { en la Ed. Parvularia }\end{array}$} & 1 año & 44,3 & 14,149 \\
\cline { 2 - 4 } & 2 años & 32,8 & 10,477 \\
\cline { 2 - 4 } & 3 años & 22,9 & 7,321 \\
\hline
\end{tabular}

Para identificar a los estudiantes de cuarto año básico que asistieron a programas de educación parvularia, se usaron las bases de datos administrativas de 2001 a 2003 de los proveedores públicos de educación parvularia (Junta Nacional de Jardines Infantiles, Junji y la Red de Salas Cuna y Jardines Infantiles, Integra). Estas bases de datos certifican la inscripción de los niños cada año e incluyen información acerca de su género y la ubicación geográfica del establecimiento educacional. Estas bases fueron facilitadas a las investigadoras por medio de convenios y protocolos de uso ético de los datos. 
a) Información sociodemográfica

El cuestionario para padres del Simce 2008 contiene información sociodemográfica, como el nivel educacional de los padres y el ingreso familiar. El Simce también incluye información acerca de la escuela, como el ingreso promedio del hogar, referencias geográficas y fuente de financiamiento (municipal ${ }^{8}$, particular-subvencionado ${ }^{9}$ y particular privado). Finalmente, el Simce incluye un cuestionario para profesores acerca de su edad, educación, certificaciones y experiencia.

El presente estudio también usó la información comunal de la Encuesta de Caracterización Socioeconómica Nacional (Casen) como la población, tasas de pobreza y tasa de empleo femenino, de manera de caracterizar el contexto social de las familias.

b) Resultados académicos

Este estudio utilizó los puntajes Simce para medir los resultados académicos. Cada año, el Simce levanta información acerca del aprendizaje de los niños en cuanto a competencias estándares en Matemáticas y Lenguaje y Comunicación, basada en el currículo nacional establecido por el Gobierno. La prueba para cuarto año básico evalúa los contenidos mínimos del primer ciclo de educación básica (primero a cuarto año). El Simce usa la Teoría de Respuesta por Ítem (IRT); en 2008, el puntaje promedio de Lenguaje fue de 260 $(D E=54)$, y el de Matemáticas fue $247(D E=55)$.

Según el reporte de Simce 2008, los niños de cuarto básico presentaron diferencias significativas de género a favor de las niñas en la prueba de Lenguaje (mujeres $=267$; hombres $=254$ ). Sin embargo, al observar la distribución de los puntajes por grupo de logro (inicial, intermedio o avanzado) se observa que en Matemáticas las niñas calificaron en una proporción significativamente menor en el grupo de logro "avanzado" (23\%) que los niños (27\%).

Escuelas públicas.

Colegios privados que reciben financiamiento estatal. 


\subsection{Estrategia analítica}

La investigación en educación de la primera infancia ha demostrado la importancia de las diferencias sociodemográficas entre los niños que asisten a la educación inicial y los que no lo hacen, es decir, la presencia de un sesgo de selección (Bainbridge, Meyers, Tanaka \& Waldfogel, 2005; Kagan, 2009). El presente estudio también muestra evidencia de este sesgo de selección en la participación en la educación parvularia. Por ejemplo, los niños chilenos que no asistieron a la educación parvularia son socioeconómicamente más diversos que el grupo de niños que sí asisten a la educación parvularia pública. Además, el grupo de niños que no asistió a educación parvularia difirió significativamente del grupo de niños que participó, lo que se refleja en indicadores como: el nivel de educación de la madre, las tasas de pobreza, el porcentaje de población viviendo en áreas rurales y el acceso a la educación parvularia pública.

Debido a las diferencias entre grupos no fue posible hacer una comparación directa para "identificar" el impacto de los programas de educación parvularia. Resultó necesario usar un procedimiento capaz de testear el impacto para grupos comparables, esto es, aquellos que cumplen con un rango similar de indicadores, difiriendo solo en su participación en la educación parvularia. Es decir, estimar el efecto de la educación parvularia mientras se controla por el sesgo de selección. Por esta razón este estudio usó Propensity Score Matching (PSM), método desarrollado por Rosenbaum y Rubin (1983).

El objetivo del PSM es parear un individuo que recibió el tratamiento con una pareja comparable que no lo recibió. Ello permite comparar niños que asistieron a la educación parvularia pública (el grupo de tratamiento) con niños que compartían características sociodemográficas similares, pero no participaron de ningún programa (el grupo control). Con ambos grupos compartiendo una probabilidad similar de haber recibido el tratamiento (Propensity Score) fue posible estimar el efecto del tratamiento (Rubin, 1997).

PSM tiene la limitación de que solo puede controlar por las variables de las que se tiene información, dejando fuera variables no observables o no medibles. En este sentido es posible que el estimador 
de PSM subestime o sobreestime los efectos del tratamiento. Para compensar esto, el presente estudio añadió tantas variables como fue posible cuando se calculó el puntaje de probabilidad (Propensity Score), con el objetivo de asegurar que el grupo de tratamiento y el de control fueran lo más similares posibles. Las variables que se utilizaron fueron las siguientes: características familiares (número de miembros y nivel de escolaridad de los padres), estadísticas del distrito (tasa de pobreza, ingreso familiar promedio, cobertura de educación parvularia, población rural y empleo femenino) y locación geográfica.

Para controlar por variables que ocurrieron una vez terminado el tratamiento (participación en educación inicial) y que también afectaron los resultados académicos, se usó un análisis de regresiones ajustado con el ponderador del Propensity Score. Por lo tanto, el efecto de la educación parvularia en los resultados académicos de cuarto básico se estimó controlando por:

- las características del niño, como el género;

- características familiares, como que el padre y/o madre cuenten con un título universitario, ingreso per cápita, número de libros en el hogar o acceso a computador e internet;

- participación en la educación parvularia; y

- determinantes a nivel de escuela, como la fuente de financiamiento, vulnerabilidad, nivel educacional y experiencia de los profesores y locación urbana o rural.

Se realizó un análisis de la muestra completa para estimar el efecto de la educación parvularia y de muestras independientes por género y cantidad de años cursados.

\section{Resultados}

\subsection{Impacto de asistir a la educación parvularia}

La Tabla 3 describe las variables de línea base para el grupo de tratamiento y de control, mientras que la Tabla 4 muestra variables específicas para las muestras pareadas y sin parear. El análisis "no pareado" compara a los niños que asistieron a la educación parvularia pública chilena con todos los niños que no lo hicieron. El 
análisis pareado comparó a los niños que sí asistieron a programas de educación parvularia con aquellos que no participaron y que compartían características sociodemográficas similares. Las columnas sin parear de la Tabla 3 muestran las diferencias sociodemográficas entre los niños que asistieron a la educación parvularia y los que no. Por ejemplo, los primeros provenían de comunas más pobres que los niños que no asistieron. La Tabla 3 también presenta el balance logrado luego de usar PSM. En la muestra ya pareada, es posible observar cómo los niños tratados y no tratados comparten características sociodemográficas, y cómo los estadísticos $t$ disminuyeron significativamente luego del pareo.

Tabla 3

Covariantes pretratamiento y estadísticos t para balance entre grupos control tratamiento

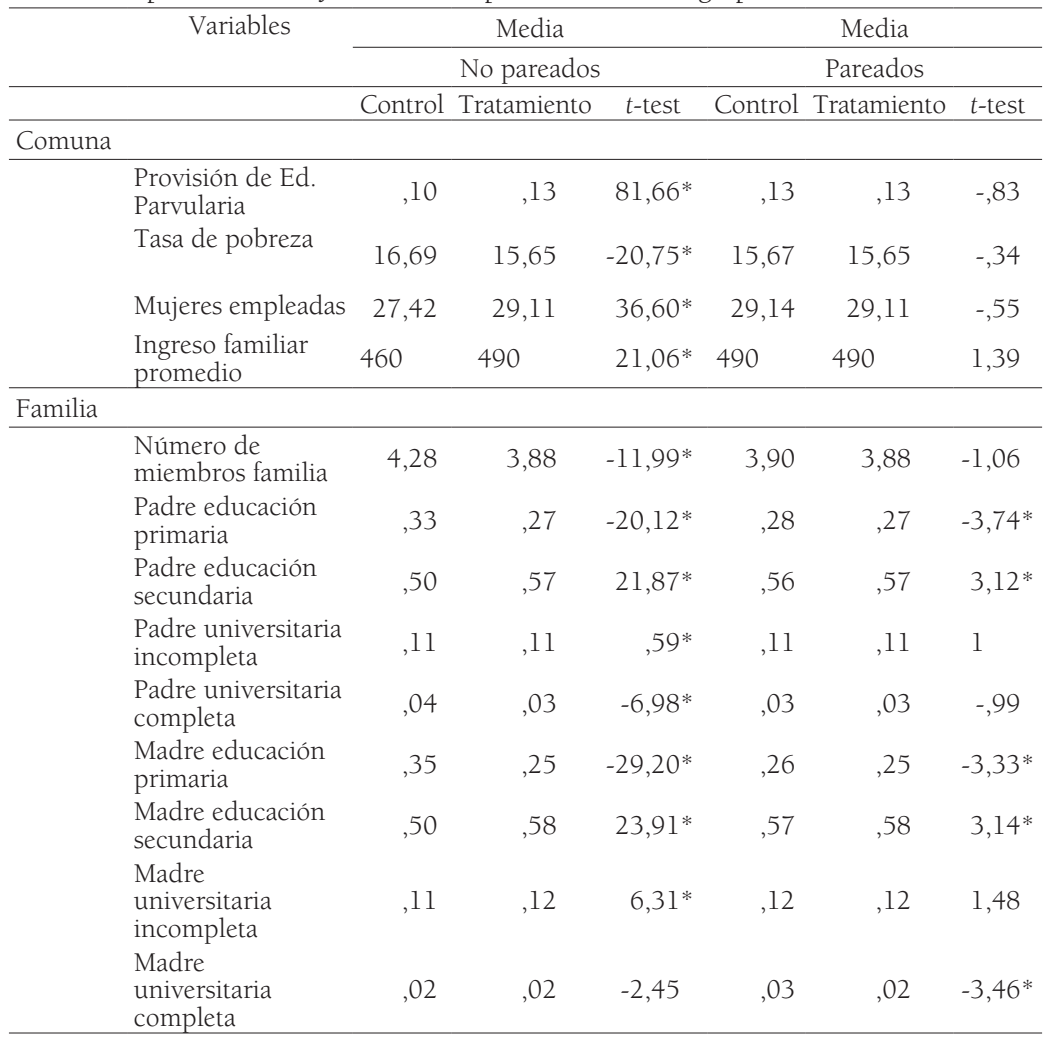

Nota. ${ }^{*} p<.01$. Educación de los padres= Porcentaje de padres que obtuvieron tal nivel. Mujeres empleadas= Porcentaje de mujeres empleadas formalmente. Ingreso promedio familiar= Monto mensual en dólares. Los grupos también fueron pareados de acuerdo a su ubicación geográfica (15 Regiones). 
La Tabla 4, en tanto, muestra los estimadores del tratamiento de acuerdo con el análisis de PSM, presentándose la diferencia de medias para el grupo de tratamiento y grupo de control de la muestra ya pareada por el PSM, seguidas por el error estándar ${ }^{10}$. Estas estimaciones muestran que la asistencia a la educación parvularia se asocia con las ganancias académicas en las tres pruebas: Matemáticas, Lenguaje y Comunicación y Ciencias Sociales. Los niños que asistieron a la educación parvularia obtuvieron en promedio puntajes superiores de 12,8 puntos (0,23 desviación estándar) en Matemáticas, y 10,7 puntos ( 0,19 desviación estándar) en Lenguaje, que los niños que no asistieron a la educación parvularia.

Los efectos de la educación parvularia y sus errores estándares derivados del modelo de regresión ajustada se muestran en las últimas dos columnas de la Tabla 4. Luego de controlar por las variables postratamiento, como se muestra en las columnas 5 y 6 , los niños que participaron en la educación parvularia obtuvieron en promedio 11,9 puntos más en Matemáticas (0,21 desviación estándar) y 9,4 puntos más en Lenguaje $(0,17$ desviación estándar) que los niños que no participaron en programas de educación parvularia. Las diferencias entre ambos análisis son pequeñas, lo cual implica que las variables postratamiento juegan un rol menor en modificar el efecto de la asistencia a programas de educación parvularia en los resultados académicos de los niños.

10 Los errores estándares para la diferencia de medias de la muestra pareada fueron calculados usando Bootstrap (1.000 respuestas). 
34 EDUCACIÓN PARVULARIA CHILENA: EFECTOS POR GÉNERO Y AÑOS DE PARTICIPACIÓN - A. Cortázar y C. Vielma

Tabla 4

Efecto de la asistencia a la educación parvularia en el logro académico

\begin{tabular}{|c|c|c|c|c|}
\hline & \multicolumn{4}{|c|}{ Muestra pareada } \\
\hline & \multicolumn{2}{|c|}{ Diferencia de medias } & \multicolumn{2}{|c|}{ Regresión ajustada PS } \\
\hline Medida de resultado & $\begin{array}{c}\text { E.T } \\
\text { (E.E) }\end{array}$ & $\mathrm{D}$ & $\begin{array}{c}\text { E.T } \\
\text { (E.E) }\end{array}$ & D \\
\hline Matemáticas & $\begin{array}{l}12,8^{*} \\
(1,3)\end{array}$ & 0,23 & $\begin{array}{l}11,9 * \\
(1,2)\end{array}$ & 0,21 \\
\hline Lenguaje & $\begin{array}{c}10,7 * \\
(1,3)\end{array}$ & 0,19 & $\begin{array}{l}9,4 * \\
(1,3)\end{array}$ & 0,17 \\
\hline Ciencias Sociales & $\begin{array}{l}9,4^{*} \\
(1,3)\end{array}$ & 0,18 & $\begin{array}{l}8,5^{*} \\
(1,2)\end{array}$ & 0,17 \\
\hline
\end{tabular}

Nota: $E . E=$ Errores estándares entre paréntesis. Errores estándares para la diferencia de medias fueron calculados con Bootstrap (1000 rps); E.T = Efecto de tratamiento; $D$ = Efecto de tratamiento en unidades de desviación estándar. Diferencias de más de 3 puntos son estadísticamente significativas.

$*=p<.05$.

\section{2. ¿Hay diferencias en el efecto de la educación parvularia según el género del niño/a?}

El efecto de la educación parvularia fue mayor para los niños que para las niñas en todas las pruebas del Simce (ver Tabla 5). Las similitudes y diferencias entre participantes y no participantes por género son similares a las descritas anteriormente para toda la muestra (ver Tabla 4).

Cuando se estimó el efecto de la educación parvularia para los hombres $(n=42.895)$ de la muestra pareada, se encontró que los niños que asistieron a la educación parvularia obtuvieron en promedio puntajes mayores: 11,5 puntos en Matemáticas $(0,2$ desviación estándar), y 6,3 puntos en Lenguaje $(0,11$ desviación estándar), en comparación con los niños que no asistieron a la educación parvularia. En el caso de las niñas $(n=43.633)$, aquellas que participaron de la educación parvularia obtuvieron un promedio de 4,9 puntos más en Matemáticas (0,08 desviación estándar) y 4,4 puntos más en Lenguaje que las niñas que no participaron de ningún programa. Las diferencias entre niños y niñas siguen presentes luego de aplicar el análisis de regresión ajustado (columnas 5 y 6 de la Tabla 5). Estos resultados sugieren que los niños se benefician más que las niñas al asistir a la educación parvularia pública en Chile. 
Tabla 5

Efecto de la participación en educación parvularia sobre el logro académico según género

\begin{tabular}{lcccc|}
\hline \multicolumn{5}{c}{ Niños } \\
\hline \multicolumn{5}{c}{ Muestra pareada } \\
\hline Medida de logro & E.T $(E E)$ & $D E$ & E.T (EE) & DE \\
\hline Matemáticas & $\begin{array}{c}11,5^{*} \\
(1,3)\end{array}$ & 0,20 & $\begin{array}{c}11,1^{*} \\
(1,2)\end{array}$ & 0,20 \\
\hline Lenguaje & $\begin{array}{c}6,3^{*} \\
(1,3)\end{array}$ & 0,11 & $\begin{array}{l}6,0^{*} \\
(1,2)\end{array}$ & 0,10 \\
\hline
\end{tabular}

\begin{tabular}{|c|c|c|c|c|}
\hline & \multicolumn{4}{|c|}{ Niñas } \\
\hline & \multicolumn{4}{|c|}{ Muestra pareada } \\
\hline & \multicolumn{2}{|c|}{ Diferencia de medias } & \multicolumn{2}{|c|}{ Regresión ajustada PS } \\
\hline Medida de logro & E.T (EE) & $D E$ & E.T (EE) & $D E$ \\
\hline Matemáticas & $\begin{array}{l}4,9^{*} \\
(1,3)\end{array}$ & 0,08 & $\begin{array}{l}4,1^{*} \\
(1,4)\end{array}$ & 0,07 \\
\hline Lenguaje & $\begin{array}{l}4,4^{*} \\
(1,3)\end{array}$ & 0,08 & $\begin{array}{l}4,2 * \\
(1,4)\end{array}$ & 0,07 \\
\hline
\end{tabular}

Nota: $E . E$ = Errores estándares entre paréntesis. Errores estándares para la diferencia de medias fueron calculados con Bootstrap (1000 rps); E. $T$ = Efecto de tratamiento; $D=$ Efecto de tratamiento en unidades de desviación estándar. Diferencias de más de 3 puntos son estadísticamente significativas.

$*=p<.05$. La significancia de la diferencia de medias (E.T) fue estimada para cada género como una muestra independiente.

\subsection{Mientras más años de educación parvularia, ¿mejor?}

Cuando se comparó a niños que asistieron a la educación parvularia por diferentes períodos de tiempo (uno, dos o tres años), las diferencias en los resultados académicos no fueron significativas, como puede observarse en la Tabla 6. Estos resultados son consistentes con los reportados por Barnett (2008) quien encontró que más educación parvularia no era necesariamente mejor. 
Tabla 6

Efecto de número de años de asistencia a la educación parvularia en el logro académico

\begin{tabular}{lcccc}
\hline Años de participación & \multicolumn{5}{c}{3 años } \\
\hline & \multicolumn{5}{c}{ Muestra pareada } \\
\hline Medida logro & Diferencia de medias & Regresión ajustada \\
\hline Matemáticas & E.T & E.E & E.T & E.E \\
& 15,7 & 1,6 & 14,4 & 1,6 \\
& $(0,28)$ & & $(0,26)$ & \\
\hline Lenguaje & 12,2 & 1,6 & 10,2 & 1,6 \\
& $(0,22)$ & & $(0,18)$ & \\
\hline
\end{tabular}

\begin{tabular}{lcccc}
\hline Años de participación & \multicolumn{4}{c}{ 2 años } \\
\hline & \multicolumn{4}{c}{ Muestra pareada } \\
\hline Medida logro & Diferencia de medias & Regresión ajustada \\
\hline Matemáticas & E.T & E.E & 12,2 & E.E \\
& 12,9 & 1,3 & $(0,22)$ & 1,4 \\
\hline Lenguaje & $(0,23)$ & & 9,1 & 1,4 \\
& 10,2 & 1,3 & $(0,16)$ & \\
\hline
\end{tabular}

\begin{tabular}{lcccc}
\hline Años de participación & \multicolumn{4}{c}{1 año } \\
\hline & \multicolumn{5}{c}{ Muestra pareada } \\
\hline Medida logro & Diferencia de medias & \multicolumn{2}{c}{ Regresión ajustada } \\
\hline & E.T & E.E & E.T & E.E \\
\hline Matemáticas & 11,8 & 1,3 & 10,8 & 1,3 \\
& $(0,21)$ & & $(0,19)$ & 1,3 \\
\hline Lenguaje & 10,2 & 1,3 & 8,5 & \\
& $(0,18)$ & & $(0,15)$ & \\
\hline
\end{tabular}

Nota: Desviaciones estándares entre paréntesis; E.T = Efecto de tratamiento; E.E = Error estándar; Grupo referencia = Todos los niños que no asistieron a la educación parvularia.

Por otro lado, en el modelo de regresión ajustada que estimó el efecto de asistir a la educación parvularia durante un año, se controló por el nivel cursado por los niños en ese año (Nivel Medio Menor, Medio Mayor y Nivel Transición 1), con el objetivo de observar los posibles efectos diferenciados de asistir a un determinado nivel. Los resultados indican que los niños que asistieron a Nivel Transición 1 obtuvieron en promedio 3 puntos más en Matemáticas y 3,5 puntos en Lenguaje, que los niños que asistieron al Nivel Medio Menor. Aunque se trata de una diferencia pequeña, se comprobó que es significativa y vale la pena considerar sus posibles causas e implicancias. En cambio, la diferencia entre haber asistido a Nivel Medio Mayor y Medio Menor no fue estadísticamente significativa. 


\section{Conclusiones}

El presente estudio encontró que los programas públicos de educación parvularia en Chile tienen un efecto de largo plazo importante sobre el logro académico de los niños medido por las pruebas Simce de cuarto año básico. Estos resultados tienen implicancias para las políticas de educación parvularia. La investigación ha demostrado que el logro académico se relaciona con el ingreso futuro del individuo, con sus posibilidades de movilidad social y, tal vez lo más importante, con sus oportunidades de ser un miembro activo de la sociedad (Programa de Promoción de la Reforma Educativa en América Latina y el Caribe, PREAL, 2006). Los programas chilenos de educación parvularia dan a los niños la oportunidad de empezar la escuela mejor preparados académicamente que sus pares que comparten características similares, pero que no asistieron a la educación parvularia. Es posible que esta ventaja facilite las vidas académicas y sociales de estos niños.

Este estudio entrega evidencia acerca de temas no explorados con anterioridad en Chile. Específicamente sobre los efectos de la educación parvularia en los resultados académicos de manera diferenciada por género y número de años en la educación parvularia.

Uno de los resultados más relevantes fue el obtenido en la dimensión de género. Aunque las posibles razones para entender este efecto diferenciado de la educación parvularia por género son variadas, una de las hipótesis es que la cultura chilena fomenta expectativas distintas para niños y niñas, las que son específicamente más exigentes para los niños. Estas expectativas pueden impactar en el comportamiento de los padres y educadoras hacia los niños pequeños, lo que se traduciría en establecer con ellos relaciones más desafiantes en lo cognitivo. Otra posible explicación es que las niñas entran a Nivel Transición 2 con mejores habilidades cognitivas (Ready et al., 2005; Soderman et al., 1999). Asumiendo que los niños entran a la educación parvularia con peores habilidades académicas que las niñas, y que los programas de educación parvularia adhieren a las mismas directrices curriculares para ambos, podría argumentarse que los niños, al comenzar la educación parvularia en desventaja, tienen más espacio para aprender en estos programas. De esta manera, las 
niñas tendrían un techo "más bajo" de aprendizaje en los programas de educación parvularia en Chile.

Por otro lado, el no haber obtenido diferencias en los resultados según años de participación en la educación parvularia no deja tampoco de ser un descubrimiento de importancia, pues sugiere que el cambio que experimentan los niños y/o las familias cuando participan de un programa de educación parvularia chileno no depende del número de años que asistan los niños, sino de la sola participación en dichos programas. La educación parvularia pública provee a los niños y a sus familias de varios servicios, tales como: estimulación cognitiva, nutrición, apoyo a la crianza y redes de apoyo. Estos programas tienen como objetivo fomentar el desarrollo del niño y su aprendizaje, ya sea de forma directa a través de un currículo de educación parvularia, como indirectamente a través del apoyo a las familias. Además, ha de relevarse el hecho de que el haber asistido un año a Nivel Transición 1 significa una mayor ganancia académica en el largo plazo, en comparación con hacerlo un año en uno de los Niveles Medios. Una posible explicación puede tener que ver con el hecho de que Nivel Transición 1 enfatiza especialmente las habilidades preacadémicas que los niveles previos de educación parvularia, además de entregar a los niños un año más de educación justo antes de entrar a Nivel Transición 2, favoreciendo la continuidad de los aprendizajes.

Estos resultados señalan áreas interesantes para investigación y políticas futuras, y ofrece una comprensión más acabada de los efectos de la educación parvularia chilena sobre el desempeño académico de los estudiantes. Los resultados de este estudio llaman a reflexionar acerca de las oportunidades de aprendizaje que se les está ofreciendo a los niños y las niñas en sus primeros años, y resaltan el desafío de considerar en los programas una educación que responda a las particularidades de desarrollo y manera de aprender tanto de los niños como de las niñas. 


\section{Referencias}

Anderson, M. (2007). Multiple inference and gender differences in the effects of preschool: A reevaluation of the Abecedarian, Perry Preschool, and early training projects. Early Childhood Research Collaborative (Discussion paper 109).

Andersson, B. (1992). Effects of day-care on cognitive and socio-emotional competence of thirteen-year-old Swedish school children. Child Development, 1(63), 20-36.

https://doi.org/10.2307/1130898

Bainbridge, J., Meyers, M., Tanaka, S., \& Waldfogel J. (2005). Who gets an early education? Family income and the enrollment of 3- to 5-yearolds from 1968-2000. Social Science Quarterly, 3(86), 724-745. https://doi.org/10.1111/j.0038-4941.2005.00326.x

Barnett, S. (1985). Benefit-cost analysis of the Perry preschool and its policy implications. American Educational Research Association, 4(7), 333-342. https://doi.org/10.2307/1163569

Barnett, S. (1995). Long-term effects of early childhood programs on cognitive and school outcomes. The Future of Children, 3(5), 25-50. https://doi.org/10.2307/1602366

Barnett, S. (2008). Preschool education and its lasting effects: Research and policy implications. Boulder and Tempe: Education and the Public Interest. Center \& Education Policy Research Unit. Recuperado de http://nepc.colorado.edu/files/PB-Barnett-EARLY-ED_FINAL.pdf

Belfield, C., Nores, M., Barnett, S., \& Schweinhart, L. (2006). The high/scope Perry preschool program. Cost-benefit analysis using data from the age- 40 follow-up. Journal of Human Resources, 1(41), 163-190. https://doi.org/10.3368/jhr.xli.1.162

Berlinski, S., Galiani, S., \& Gertler, P. (2006). The effect of pre-primary education on primary school performance (IFS Working Papers W06/04). London: Institute for Fiscal Studies.

Berlinski, S., Galiani, S., \& Manacorda, M. (2008). Giving children a better start: Preschool attendance and school-age profiles. Journal of Public Economics, 5(92), 1416-1440.

https://doi.org/10.1016/j.jpubeco.2007.10.007

Besharov, D., Germanis, P., Higney, C., \& Call, D. (2011). Assessing the evaluations of early childhood education programs: Chapter 16. The High/Scope Perry Preschool Project. Welfare Reform Academy. Recuperado de http://www.welfareacademy.org/pubs/early_education/pdfs/Besharov_ ECE\%20assessments_Perry_Preschool.pdf 
Bucarey, A., Urzúa, S., y Ugarte, G. (2014). El efecto de la educación preescolar en Chile (Documento de trabajo). Santiago: Universidad de Chile.

Camilli, G., Vargas, S., Ryan, S., \& Barnett, W. S. (2010). Meta-analysis of the effects of early education interventions on cognitive and social development. Teachers College Record. Recuperado de http://www.tcrecord.org/Content.asp?ContentID=15440

Campbell, F, Pungello, E., Miller-Johnson, S., Burchinal, M., \& Ramey, C. (2001). The development of cognitive and academic abilities: Growth curves from an early childhood educational experiment. Developmental Psychology, 2(37), 231-242.

https://doi.org/10.1037//0012-1649.37.2.231

Caughy, M., Di Pietro, J., \& Strobino, D. (1994). Day-care participation as a protective factor in the cognitive development of low-income children. Child Development, 2(65), 457-471. https://doi.org/10.2307/1131396

Contreras, D., Herrera, R., y Leyton, G. (2007). Impacto de la educación preescolar sobre el logro educacional: Evidencia para Chile [The impact of early childhood education on academic achievement: Evidence for Chile]. Santiago de Chile: Universidad de Chile.

Eyzaguirre, B. y Le Foulon, C. (2001). La calidad de la educación chilena en cifras. Estudios Públicos, 84, 85-204.

García, J. L., Heckman, J. J., \& Ziff, A. L. (2017). Gender differences in the benefits of an influential early childhood program (NBER Working Paper No. 23412). Chicago: University of Chicago.

Heckman, J. (2000). Invest in the very young (Working paper). Chicago, IL: The Harris School, University of Chicago.

Hernández, P. (2006). Impacto de la asistencia a educación preescolar en logros académicos posteriores: el caso chileno (Tesis de Magíster en Economía Aplicada, Universidad de Chile, Santiago, Chile).

Kagan, S. (2009). American early childhood education: Preventing or perpetuating inequity (Equity matters: Research review No.3). New York: Campaign for Educational Equity, Teachers College.

Magnuson, K., Meyers, M., Ruhm, C., \& Waldfogel, J. (2004). Inequality in preschool education and school readiness. American Educational Research Journal, 1(41), 115-157. https://doi.org/10.3102/00028312041001115

Magnuson, K., Ruhm, C., \& Waldfogel, J. (2007). The persistence of preschool effects: Do subsequent classroom experiences matter? Early Childhood Research Quarterly, 22, 18-38.

https://doi.org/10.1016/j.ecresq.2006.10.002 
McKay, A. \& McKay, H. (1983). Primary school progress after preschool experience: Troublesome issues in the conduct of follow-up research and findings from the Cali, Colombia Study. En K. King \& R. Myers (Eds.), Preventing school failure (pp. 36-41). Ottawa, CN: International Development Research Center.

Ministerio de Educación de Chile, Mineduc (2013). El estado del arte de la educación parvularia. Recuperado de http://redlacti.com/php/biblioteca/ups/1393257789.pdf

Ministerio de Planificación y Cooperación, Mideplan (2011). Encuesta de caracterización socio-económica nacional-CASEN Primera Infancia. Santiago de Chile: Autor.

National Institute of Child Health and Human Development Early Child Care Research Network, NICHD (2005). Duration and developmental timing of poverty and children's cognitive and social development from birth through third grade. Child Development, 4(76), 795-810. https://doi.org/10.1111/j.1467-8624.2005.00878.x

Nelson, G., Westhues, A., \& MacLeod, J. (2003). A meta-analysis of longitudinal research on preschool prevention programs for children. Prevention and Treatment, 1(6), 1-67. https://doi.org/10.1037/1522-3736.6.1.631a

Nores, M. \& Barnett, S. (2010). Benefits of early childhood interventions across the world: (Under) investing in the very young. Economics of Education Review, 2(29), 271-282.

https://doi.org/10.1016/j.econedurev.2009.09.001

Organisation for Economic Co-operation and Development, OECD. (2013). Education at a Glance 2013. OECD Indicators. Paris: OECD Publishing.

Pacheco, P., Elacqua, G., y Brunner, J. (2005). Educación preescolar estrategia bicentenario. Recuperado de www.oei.es/inicial/politica/estrategia_bicentenario_chile.pdf

Programa de Promoción de la Reforma Educativa en América Latina y el Caribe, PREAL. (2006). Educación y brechas de equidad en América Latina. Volumen II. Santiago de Chile: Editorial San Marino.

Ramey, C. \& Ramey, S. (2006). Early learning and school readiness: Can early intervention make a difference? En N. Watt, C. Ayoub, R. Bradley, J. Puma, \& W. Lebeouf (Eds.), The crisis in youth mental health: Critical issues and effective programs: Vol. 4 Early intervention programs and policies (pp. 291-317). Westport: Praeger Press.

Ready, D., LoGerfo, L., Brukham, D., \& Lee, V. (2005). Explaining girls' advantage in kindergarten literacy learning: Do classroom behaviors 
make a difference? The Elementary School Journal, 1(106), 21-38. https://doi.org/10.1086/496905

Reveco, O. y Mella, O. (1999). El impacto de la educación parvularia en la educación básica. Recuperado de www.isharon.files.wordpress.com/2007/06/impacto-de-la-educacionparvularia-en-la-educacion-basica.pdf

Rosenbaum, P. \& Rubin, D. (1983). The central role of the propensity score in observational studies for causal effects. Biometrika, 1(70), 41-55. https://doi.org/10.2307/2335942

Rubin, D. (1997). Estimating causal effects from large data sets using propensity score. Annals of Internal Medicine, 8(127), 757-763. https://doi.org/10.7326/0003-4819-127-8_part_2-199710151-00064

Shonkoff, J. P. \& Phillips, D. A. (Eds.) (2000). From neurons to neighborhoods: The science of early child development. Washington, DC: National Academy Press.

Shore, R. (1997). Rethinking the brain: New insights into early development. New York: Families and Work Institute.

Siegel, D. (1999). The developing mind. New York: Guilford Press.

Soderman, A., Chhikara, S., \& Kuo, E. (1999). Gender differences that affect emerging literacy in first grade children: The U.S., India, and Taiwan. International Journal of Early Childhood, 2(31), 9-16. https://doi.org/10.1007/bf03166892

United Nations Educational, Scientific and Cultural Organization, Unesco (2010). Global education digest. Comparing education statistics across the world. Montreal: Unesco, Institute for Statistics.

Vandell, D. \& Corasaniti, M. (1990). Variations in child care: Do they predict subsequent social, emotional and cognitive differences? Early Childhood Research Quarterly, 4(5), 555-572. https://doi.org/10.1016/0885-2006(90)90019-w

Recibido: 22/03/2017

Aceptado: 17/07/2017 